\title{
Machine Learning Algorithms for Human Activity Recognition
}

\author{
K.R.Baskaran, M.N.Saroja
}

\begin{abstract}
It becomes essential to monitor the Activity of Daily Living $(A D L)$ of elderly people living alone by keeping track of their day to day activities \& helping those having strong health issues. In this paper various machine learning algorithms for human activity recognition is analyzed. Along with this, an extensive study is carried out to learn about the current technologies used in activity recognition. Activity recognition is generally done in the form of signals generated through sensors. The signals are then preprocessed, segmented, features are extracted and activity is recognized. The main objective of Human Activity Recognition System is to explore the limitations of self-dependent old age persons and suggest ways of overcoming it. By using the different wearable and non-wearable sensors, one can easily monitor the human activity and evaluate the data generated through it.
\end{abstract}

Index Terms- Machine learning, Human Activity Recognition, Hidden Markov Model.

\section{INTRODUCTION}

Activities of daily living (ADL) is used to monitor the routine tasks and status of aged people living alone. Activity recognition also plays a major role in healthcare monitoring and surveillance systems. Monitoring and evaluating the day-to-day activities is not viable in real life context [2].In household appliances, sensors are used to monitor one's activity efficiently.

Assisted living systems (ALSs) try out the most additive way to help elder people's daily life such as healthcare approaches through the wearable and non-wearable sensors. They fix up with the advanced technique to reform the selfreliant elder people's independent living.

Ambient-sensor-based ALSs are equipped with both the multiple ambient sensors (wearable) and non-wearable sensors in the room or on some specific objects, such as temperature sensors, passive infrared sensors, pressure sensors, door switches, etc. [1]. No single type of assisted living system sensors are used practically due to their nonadaptive characteristics.

The recent research area that faces lot of challenges is human activity recognition field that focuses on mainly health care applications and some applications regarding security issues. In human activity monitoring, to detect the motion and objects, computer vision-based techniques are used [15]. Along with this, they mainly commit with the infrastructure. For example, monitoring the areas through the video camera setup. To replace this, track one's motion using worn sensors or track via sensors fixed in one's smartphone [12].

Table 1: Types of Activities Recognized By HAR System

\begin{tabular}{|l|l|}
\hline ACTIVITIES & GROUPS \\
\hline $\begin{array}{l}\text { Eating, drinking, working at the } \\
\text { PC, watching TV, reading, } \\
\text { brushing teeth. }\end{array}$ & $\begin{array}{l}\text { Daily } \\
\text { activities }\end{array}$ \\
\hline $\begin{array}{l}\text { Riding a bus, cycling and } \\
\text { driving. }\end{array}$ & Transportation \\
\hline
\end{tabular}

Likewise, RFID technology is also used in human activity recognition. This system consists of antennas, readers and tags. The signal send through the antenna and tag receives the signal [6], with its identification number the signal send back to the antenna.

\section{HUMAN ACTIVITY RECOGNITION SYSTEM}

In Human activity Recognition (HAR), the aged people daily activities tracked in smart homes also. In activity recognition, sensor plays a major role. Sensors monitor and track the information from human indications, evaluate the information through recognition engine and analyse the different types of activities. Many sensors are attached to human body to collect the data and vital rate like pulse, temperature and respiratory rates [13]. Wrist worn accelerometer, 3D-axial accelerometer, magnetometer, and RFID tag are the widely used sensors in activity recognition. Currently with the pervasiveness of smart phones, they have inbuilt accelerometer, magnetometer and gyroscope to sense the user. Activity recognition has two stages as other machine learning algorithms. They are training and testing or it also called as evaluation.

HAR systems are classified based on wearable sensing techniques in two levels. One is done with the learning approach; it can be either supervised or semi-supervised. It is diagrammatically represented in Figure 1. Supervised approaches can work either online or offline according to the requirement needed. Online Supervised HAR system is an application that easily project activity recognized from human. Offline Supervised HAR System is an application that is used in offline, where in some cases the user has no need of an immediate reply [10]. Semi-supervised systems is in between label and unlabelled data.
Revised Manuscript Received on September 14, 2019.

Dr.K.R.Baskaran, Professor, Department of Computer Science and Engineering, Kumaraguru College of Technology, Coimbatore, TamilNadu, India.

M.N.Saroja, Assistant Professor, Department of Information Technology, Kumaraguru College of Technology, Coimbatore, TamilNadu, India.. 


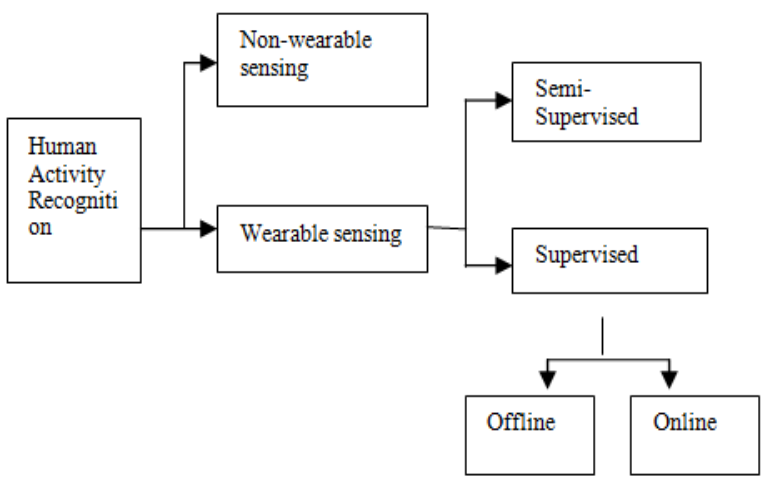

Figure 1: Taxonomy of HAR system

As stated in Figure 2, collect the data from the sensors. To measure acceleration, tri-axial accelerometer is used and data has $\mathrm{x}$-axis, $\mathrm{y}$-axis and z-axis. They capture the user's movements. Here X-axis indicates sideway or horizontal movement of the user, Y-axis indicates upward or downward movement and Z-axis indicates forward or backward movement of the user [3]. Then the informative features from the data is extracted. To evaluate the features on activity recognition, the training data are used. To improve the performance and quality, clustering method is used.

In data collection, it collects the data from both training and testing dataset, and in testing the data collected through window time. Then the structural and statistical features are extracted. Finally it recognizes the physical activity and it models.

The data generated from accelerometer is time series data and it is denoted by AX, AY AND AZ along X, Y and Zaxis respectively. A technique that separates $\mathrm{AC}$ component from the DC component between every time interval is digital low pass filter.

A noticeable and real time implementation framework helps to satisfy the general needs of elderly people [11].Wearable sensors help in developing new applications. The following aspects are used to evaluate the HAR system.

- $\quad$ Selection of sensors

- Data collection and Clustering of Data

- Feature extraction method(s)

- Learning algorithm(s)

- $\quad$ Recognizing model and activity

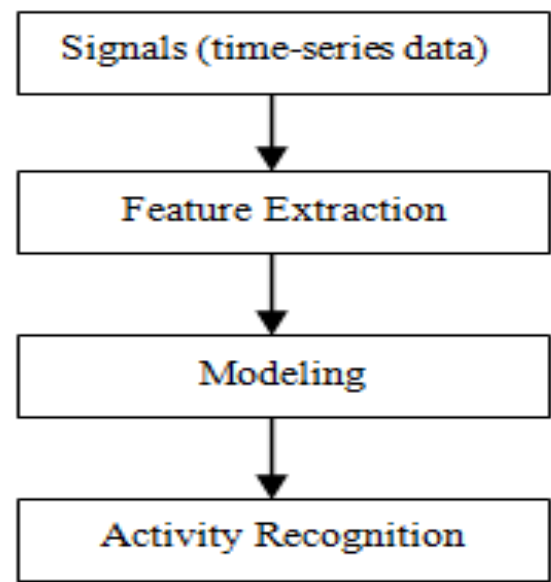

Figure 2: Flowchart of HAR

\section{MODELS USED FOR ACTIVITY RECOGNITION \& RESULTS}

This section represents the HMM model, Artificial Neural Network and Dictionary Learning Algorithm for human activity recognition. These methodologies will be useful and effective in user's activity recognition.

\section{A. Hidden Markov Model (HMM)}

Hidden Markov Model plays a major role in activity recognition system. It is used to recognize the gesture, speech and pattern recognition. This paper represents the techniques that are used in activity recognition and it classifies the data along with time instances [8].

Activities can be analyzed through the set of detected data from the sensors. Collection of data can be done by using wearable sensors and image processing technique. For example, wearing Accelerometer on one's body can collect the data and overcome from some constraints such as flexibility and privacy [9].

The HMM based activity recognition can be outlined as,

- Description about the gestures

- Collection of training data

- $\quad$ Tracking the HMM through the trained (raw) data and evaluating it

It monitors the activities such as walking and sleeping. By using wearable sensors, it collects testing and training data. From the tri-axial accelerometer and gyroscope sensors, it collects and sends the data to feature extraction. In feature extraction, it has pre-processed data from the sensor output and it recognizes the activities from processed data like walking upstairs, lying, sitting etc. According to the recognized activity the model works.

HMM analyse and explore the various states based on various human activities. Analysing the activities can be done by temporal patterns. The workability of the developed model is evaluated by testing stage [5].

The key technique Posture classification is widely used for analysing the activities of people. So HMM brings out the self-regulating activity recognition and human detection. Before HMM module, Pre-processing is done by the trained datasets. Gaussian mixture model is used to perform Background Subtraction.

\section{B. Artificial Neural Networks}

Neural Networks are used for recognizing the daily activities of a human. The main challenge in designing an artificial neural network for HAR is to find out the number of hidden layers. 2-layer feed forward network can be used in case of HAR. Also, Concurrent Neural Network and Recurrent Neural Networks, the widely prevalent deep learning techniques can be used to optimize the problem of HAR.

\section{Dictionary Learning Algorithm}

Dictionary Learning Algorithm plays a main role in signal processesing and in machine learning areas. As Human Activity Recognition needs input from sensor signals, dictionary learning algorithm will be best suited for this

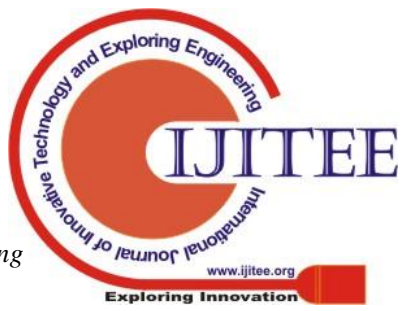


case. Creating dictionary from a large set of input signals and using them in analyzing is easier. It involves two stages coding stage and dictionary update stage.

The algorithm also works fine in both offline and online stages. Offline stages involves collecting data from sensors and then processing it. Online stage updates the data whenever there is an arrival of a new signal.

To extract the time series data, two methods are used: structural and statistical. In structural, it describes the correlation with the data and in statistical, the Fourier and Wavelet transform extract the features by quantitative characteristics [4]. It represents the flexibility and processing stages of data compared to MOD, K-SVD.

In real time, recognizing the activities is not workable. Due to pre-processing the efficiency and flexibility of models are upgraded. A productive Dictionary learning algorithm is proposed to overcome the problem and to provide a practical solution [7]. By using the below mentioned models, human activities can be easily understood

- $\quad$ Support Vector Machines (SVM)

- Instance based learning (IBL)

- Bayesian methods

- $\quad$ Ensembles of classifiers

- Decision trees

Wearable sensors provide the cost efficient, recognition performance, and exact solution for the human activity recognition. It can be applied in supervision and identification systems [14].

Table 1: HMM and Dictionary Learning Algorithm comparison

\begin{tabular}{|l|l|l|l|}
\hline Method & Activities & Advantage & Disadvantage \\
\hline HMM & $\begin{array}{l}\text { Speech } \\
\text { recognition, } \\
\text { Gesture } \\
\text { recognition }\end{array}$ & $\begin{array}{l}\text { The data from } \\
\text { wearable sensors } \\
\text { in HMM can be } \\
\text { used effectively } \\
\text { for Speech } \\
\text { Recognition. }\end{array}$ & $\begin{array}{l}\text { It has 3 issues: } \\
\text { Evaluating problem, } \\
\text { Decoding problem } \\
\text { and Learning } \\
\text { problem }\end{array}$ \\
\hline $\begin{array}{l}\text { Lictionary } \\
\text { Aearning } \\
\text { Algorithm }\end{array}$ & $\begin{array}{l}\text { Sitting down } \\
\text { on and a } \\
\text { chair(SDC), } \\
\text { standing up } \\
\text { from a chair } \\
\text { (SUC), } \\
\text { Walking }\end{array}$ & $\begin{array}{l}\text { Tests of the } \\
\text { sensors are } \\
\text { minimum and it } \\
\text { achieves high } \\
\text { accuracy. }\end{array}$ & $\begin{array}{l}\text { Recognition of more } \\
\text { challenging reiterated } \\
\text { and composite } \\
\text { actions in human } \\
\text { behaviour efficiency } \\
\text { is less. }\end{array}$ \\
\hline $\begin{array}{l}\text { Artificial } \\
\text { Neural } \\
\text { Network }\end{array}$ & $\begin{array}{l}\text { Arm posture } \\
\text { recognition, } \\
\text { Body posture } \\
\text { recognition }\end{array}$ & $\begin{array}{l}\text { Effective in } \\
\text { recognising } \\
\text { static and } \\
\text { dynamic } \\
\text { activities }\end{array}$ & $\begin{array}{l}\text { Computational } \\
\text { complexity will be } \\
\text { more if the number } \\
\text { of layers are } \\
\text { increased }\end{array}$ \\
\hline
\end{tabular}

\section{CONCLUSION AND FUTURE WORK}

This paper represents the activity recognition of aged people and monitor their activities through HMM model, ANN, Dictionary Learning Algorithm, wearable and nonwearable sensors. There are some drawbacks in current system like privacy as well as efficient tracking and accuracy. In future work, it is proposed to explore various models and its efficiency through more sensor data extracted from different physical activities.

\section{REFERENCES}

1. Pubali De , Amitava Chatterjee, and Anjan Rakshit ," Recognition of Human Behavior for Assisted living using Dictionary Learning Approach", IEEE SENSORS JOURNAL, VOL. 18, NO. 6, MARCH 15, 2018

2. Uriel Martinez-Hernandez, Imran Mahmood, and Abbas A. Dehghani-Sanij,"Simultaneous Bayesian Recognition of Locomotion and Gait Phases With Wearable Sensors", IEEE SENSORS JOURNAL, VOL. 18, NO. 3, FEBRUARY 1, 2018

3. Akram Bayat, Marc Pomplun, Duc A. Tran, "A Study on Human Activity Recognition Using Accelerometer Data from Smartphones", ELSEVIER 2014

4. Bo Tang, Jin Xu, Haibo Heand Hong Man," ADL: Active Dictionary Learning for Sparse Representation", IEEE 2017.

5. Eunju Kim, Sumi Helal, and Diane Cook," Human Activity Recognition and Pattern Discovery", IEEE 2010.

6. Christian Debes, Andreas Merentitis, Sergey Sukhanov,Maria Niessen, Nicolaos Frangiadakis, and Alexander Bauer, "Monitoring Activities of Daily Living in Smart Homes", IEEE 2016.

7. Meisam razaviyayn hung-wei tseng zhi-quan luo, "Dictionary learning for sparse representation: complexity and algorithms", IEEE international conference 2014.

8. Shagun Shaily, Veenu Mangat, "The Hidden Markov Model and its Application to Human Activity Recognition", IEEE 2015.

9. Palwasha Afsar, Paulo Cortez, Henrique Santos, "Automatic Human Action Recognition from Video using Hidden Markov Model", IEEE 2015.

10. Rong liu, ting chen, lu huang," Research on human activity recognition based on active learning", IEEE 2010.

11. Yan Wang, Shuang Cang2, Hongnian Yu1, "A noncontact-sensor surveillance system towards assisting independent living for older people", Research gate, December 2017.

12. Liming Chen, Member, IEEE, Jesse Hoey, Chris D. Nugent, Member, IEEE, Diane J. Cook, and Zhiwen Yu, "Sensor-Based Activity Recognition", IEEE, VOL. 42, NO. 6, NOVEMBER 2012

13. O' scar D. Lara and Miguel A. Labrador, "A Survey on Human Activity Recognition using Wearable Sensors", IEEE communications surveys \& tutorials, 2013.

14. Ong Chin Ann, Lau Bee Theng, "Human Activity Recognition: A Review", 2014 IEEE International Conference on Control System, Computing and Engineering, 28 - 30 November 2014.

15. Siddharth S, Rautaray, Anupam Agrawal, "Vision based hand gesture recognition for human computer interaction: a survey", November 2012 Springer science. 